Terr. Atmos. Ocean. Sci., Vol. 18, No. 3, 623-642, August 2007

\title{
The Role of Cloud Radiative Forcing in the Asian-Pacific Summer Monsoon
}

\author{
Chi-Hua Wu ${ }^{1}$ and Wen- Shung Kau ${ }^{1}$, * \\ (Manuscript received 28 March 2006, in final form 8 February 2007)
}

\begin{abstract}
Convective-cloud clusters with strong precipitation occur frequently in most of the Asian-Pacific summer monsoon (APSM) regions such as the Bay of Bengal (BOB), South China Sea (SCS), and Tropical Western North Pacific (TWNP). Cloud radiative forcing (CRF) is important in these regions. The net CRF at the top of the atmosphere (TOA) has shown large cooling over these APSM regions. This is on account of the presence of large amounts of high clouds with large optical depth. Through data analysis, the summer convective precipitation in TWNP is as strong as that in the BOB. However, the average net CRF at the TOA in the BOB $\left(\sim-36 \mathrm{Wm}^{-2}\right)$ is twice as big as in the TWNP $\left(\sim-17 \mathrm{Wm}^{-2}\right)$. The spectral analysis of cloud optical depth shows that in the $\mathrm{BOB}$, the highest power is in the intra-seasonal timescale, while in the TWNP, the leading spectral peaks are less than 10 days. The radiative cooling from net $\mathrm{CRF}$ at the TOA could be associated with lowfrequency oscillation. The difference between the APSM regions is related to their sub-stages separating from CRF in time evolution.

In a convective system, convective clouds can detrain to form other high clouds. In the APSM regions, large areas of high-thin and high-thick clouds cause different CRF at the TOA. These two types of CRF relate to precipitation, atmospheric vertical motion, and cloud life cycles etc. and should be separated from the APSM time evolution. We divided the APSM precipitation into two categories. As in the heavy-precipitation stage, clouds with large optical depth shield solar radiation and cause local and instantaneous surface cooling. The outgoing longwave radiation (OLR) is generally lower than $210\left(\mathrm{Wm}^{-2}\right)$. The net CRF at the TOA is large negatively.
\end{abstract}

\footnotetext{
${ }^{1}$ Department of Atmospheric Sciences, National Taiwan University, Taipei, Taiwan, ROC

* Corresponding author address: Prof. Wen-Shung Kau, Department of Atmospheric Sciences, National Taiwan University, Taipei, Taiwan, ROC; E-mail: wen@atmos1.as.ntu.edu.tw doi: 10.3319/TAO.2007.18.3.623(A)
} 
Besides, large high-thin clouds can be found in a stage of relatively small or no precipitation. The OLR in this stage has a broad range and the net CRF is small and could be either positive or negative. The major difference between the APSM regions occurs in this stage. In this stage at the BOB, significant high-thick clouds cause negative net $\mathrm{CRF}$, while more than half of the SCS and TWNP at this stage is dominated by large amounts of cirrus clouds. The optically thin cirrus clouds with large spatial size and long lasting time are important modulators for modifying the net CRF at the TOA. The poor simulation of the APSM climate in general circulation models (GCMs) maybe associated with the inability for accurately simulating the role of cirrus clouds in this stage.

Based on the cloud classification of the International Satellite Cloud Climatology Project (ISCCP), we found a useful cloud-amount index from cloud amounts of cirrus minus the sum of deep convection and cirrostratus. The index can effectively separate different characteristics of CRF from the APSM time evolution. The cloud-amount index should be more appropriate for APSM studies and model simulations instead of considering only one cloud type in convective systems.

(Key words: Asian-Pacific Summer Monsoon, Cloud Radiative Forcing, GCMs)

\section{INTRODUCTION}

Clouds are important climate modulators. Organized convection in tropical mesoscale convective systems spans from 10 to $100 \mathrm{~km}$ (cloud clustering), up to $1000 \mathrm{~km}$ (in the stratiform region) (Houze 1993, 2004). Clouds also trace in time to reveal "time clusters", which spatially overlap from one frame of imagery to the next. In some cases, these time clusters can last for more than two days (Mapes and Houze 1993) and are apparently real physical entities for a changing climate. The cloud radiative forcing $(\mathrm{CRF})$ at the top of the atmosphere (TOA) is significantly negative in the Asian-Pacific summer monsoon (APSM). This is a unique characteristic of the APSM (Rajeevan and Srinivasan 2000). In other words, the CRF is affected by convective systems that cool the energy budget in the APSM regions.

The Indian summer monsoon (ISM) and western North Pacific summer monsoon (WNPSM) are major monsoon systems in the APSM (Lin and Wang 2002; Wang and Lin 2002). Both of the ISM and WNPSM have complex convective systems and with different convection size, strength and coverage etc. In the whole summer, the cloud system in ISM and WNPSM is apparently a super cloud cluster (Mapes and Houze 1993; Lau and Sui 1997), which is affected by the monsoon gyre (Lander 1994), subtropical high and the intraseasonal oscillation etc. The movement of these cloud clusters associates with a shift in the major convection center from the Indian Ocean to the open ocean east of the Philippine Islands (Lin and Wang 2002). Besides, cloud distribution in the WNPSM has significant scale dependence. It is a confluence region between the monsoonal southwesterlies and the trade easterlies, and tropical 
cyclone formation is common here (Frank 1982; Briegel and Frank 1997; Ritchie and Holland 1999). Discussing cloud information is more complex in the WNPSM than in ISM.

The CRF is generally defined as the difference between radiative fluxes with and without clouds. The magnitude of net CRF (NCRF), which is defined as the sum of shortwave CRF (SWCRF) and longwave CRF (LWCRF) (Hartmann and Doelling 1991), is near cancelled in the tropical regions at the TOA. In convective systems, the convective clouds and anvil clouds are expansive and interconnected. Thus the observed CRF generally result from more than two cloud types. Many general circulation model (GCM) studies have shown that the CRF strengthens precipitation and circulation patterns and suggest that it must be parameterized accurately to simulate the tropical atmosphere (Ramanathan et al. 1983; Slingo and Slingo 1988; Randall et al. 1989; Ma et al. 1996). Indeed, as shown in Rajeevan and Srinivasan (2000), some GCMs do not simulate the mean structure of the Asian monsoon correctly because of their inability in correctly simulating CRF.

Jakob (2003) pointed out that one of the major model errors in most convective active regions on Earth is caused by errors in times (frequently observed) when deep convection is suppressed. This is a critical problem when dealing with cloud effects especially in the ISM and WNPSM regions, which are generally dominated by high clouds (Kau et al. 2003) associated with convective systems. Traditional cloud schemes have more than one type of cloud information. For example, in the cloud prediction scheme developed by Slingo and Slingo (1991), the convective cloud amount is found from the instantaneous precipitation rate. Cirrusanvil clouds are predicted if the convective cloud amount is larger than 30\% (Slingo and Slingo 1991). However, realistically high-thin clouds and their effects in the APSM regions are not clear.

High clouds with thin optical depth such as cirrus (ice clouds) have a major effect on the earth's radiation balance and climate (Liou 1986). Large amounts of cirrus increase LWCRF at the TOA and warm the atmosphere (Cox 1971). The moisture source of cirrus generally connects to convective systems. However, Luo and Rossow (2004) consider detrainment cirrus as being distinct from in situ cirrus because more than half of cirrus clouds forming in situ develop well away from convection in tropical regions. Over the APSM regions, high clouds occur all summer (Kau et al. 2003) regardless of convection (though they maybe caused by the short life cycle of convection). Positive NCRF dominated by high-thin clouds is different to the negative NCRF associated with convective clouds. Fu et al. (1990) used a combination of visible-infrared and infrared-only threshold methods to analyze the properties of convective systems. They were successful in isolating convective clouds and their characteristics.

We suggest that an effective classification for cloud effects can simplify the complexity of clouds both in terms of CRF and latent-heat release. Separation from CRF in large-scale effects of convective systems could be more useful in the APSM regions, and is easier than dealing with detailed cloud microphysical processes. After a brief description of the data and cloud information in Section 2, we will explore the properties of separation from CRF in the APSM regions in Section 3. We show major separation from the convective properties and discuss different CRF with precipitation. In Section 4, we will discuss the potential role of CRF in each APSM region especially the difference between APSM regions. Our discussion and summary are in Sections 5 and 6. 


\section{DATA}

The precipitation data used in this study is daily mean temporal resolution and $1^{\circ} \times 1^{\circ}$ in spatial resolution from the 3B42 type of the Tropical Rainfall Measuring Mission (TRMM) (Kummerow et al. 1998). The dynamic field is from version 2 of the National Centers for Environmental Prediction reanalysis data (NCEP-R2) (http://wwwt.ncep.noaa.gov/). The radiation data we used include daily mean outgoing longwave radiation (OLR) $\left(2.5^{\circ} \times 2.5^{\circ}\right.$ in spatial resolution) which is analyzed from the European Center for Medium-Range Weather Forecasts (ECMWF) reanalysis products (http://www.ecmwf.int/), and the monthly mean CRF data from the International Satellite Cloud Climatology Project (ISCCP) FD type at the TOA (Rossow and Dueñas 2004).

Major cloud information is from ISCCP-D1 type data (Rossow and Garder 1993; Rossow and Schiffer 1999). It provides 3-hourly cloud parameters (we combine them to be daily-mean data) such as cloud height, cloud optical depth and cloud amount from a global network of geostationary weather satellites and at least one polar orbiting satellite. The ISCCP cloud classification includes 9 types based on cloud optical depth and cloud top pressure as shown in Fig. 1. The mean cloud top pressure of high clouds (the major cloud types in this study) in ISCCP is lower than $440 \mathrm{mb}$ and includes three types: cirrus (CI), cirrostratus (CS), and deep convection (DC). These cloud types form by clouds which not only interconnect with each other but also feedback into the climate individually or through their communities. The cloud coverage (or more correctly hydrometeor cover, since the radar is sensitive to both cloud and precipitation size particles) of ISCCP-D1 type is defined only over a large area $(280 \times 280 \mathrm{~km})$. The use of point observations to characterize the clouds is limited to making statistical assessments. Besides, the ISCCP analyze infrared and visible radiances measured by the imaging instruments on all of the operational weather satellites. Thus parts of the results are restricted to local daytime since the satellite channels in the visible part of the spectrum data.

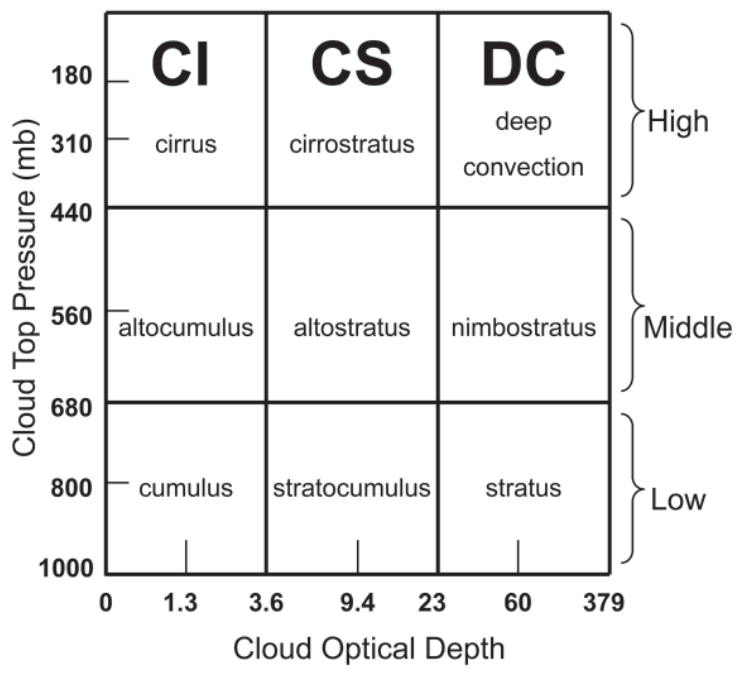

Fig. 1. ISCCP cloud-type map (re: cloud top pressure and cloud optical depth). 
In following analysis, we use the daily-mean data in an area mean of $10^{\circ} \times 10^{\circ}$ and the monthly-mean data in an area mean of $5^{\circ} \times 5^{\circ}$ for reducing uncertainty of cloud coverage during $1998 \sim 2000$ summertime (JJA). The major regions are the Bay of Bengal (BOB: $10^{\circ} \mathrm{N}$ $\sim 20^{\circ} \mathrm{N}, 85^{\circ} \mathrm{E} \sim 95^{\circ} \mathrm{E}$ ), South China Sea (SCS: $10^{\circ} \mathrm{N} \sim 20^{\circ} \mathrm{N}, 110^{\circ} \mathrm{E} \sim 120^{\circ} \mathrm{E}$ ), and Tropical Western North Pacific (TWNP: $10^{\circ} \mathrm{N} \sim 20^{\circ} \mathrm{N}, 125^{\circ} \mathrm{E} \sim 135^{\circ} \mathrm{E}$ ). The BOB belongs to the ISM and the TWNP is a typical WNPSM region. We also analyze the SCS between the BOB and the TWNP. All of these oceanic regions are at the same latitude and affected by monsoonal circulation. We define these regions as the APSM regions in this study.

\section{SEPARATION FROM CLOUD RADIATIVE FORCING}

Figure 2 shows the summer NCRF pattern (with explicit value) at the TOA and three major analyzed regions in the APSM. As shown in Fig. 2, significant negative NCRF can be found in the APSM regions. The APSM monsoonal circulation produces cloud systems and cools the climate system by their CRF. The summer NCRF in the BOB $\left(\sim-36 \mathrm{Wm}^{-2}\right)$ is near twice as big as in the SCS $\left(\sim-20 \mathrm{Wm}^{-2}\right)$ and the TWNP $\left(\sim-17 \mathrm{Wm}^{-2}\right)$. The time-space structures of the APSM play important roles in these differences. The strong-dynamic forcing results in a significant negative NCRF in the BOB. However a brief recess of convective events occurs

$1998 \sim 2000$ JJA TOA NCRF

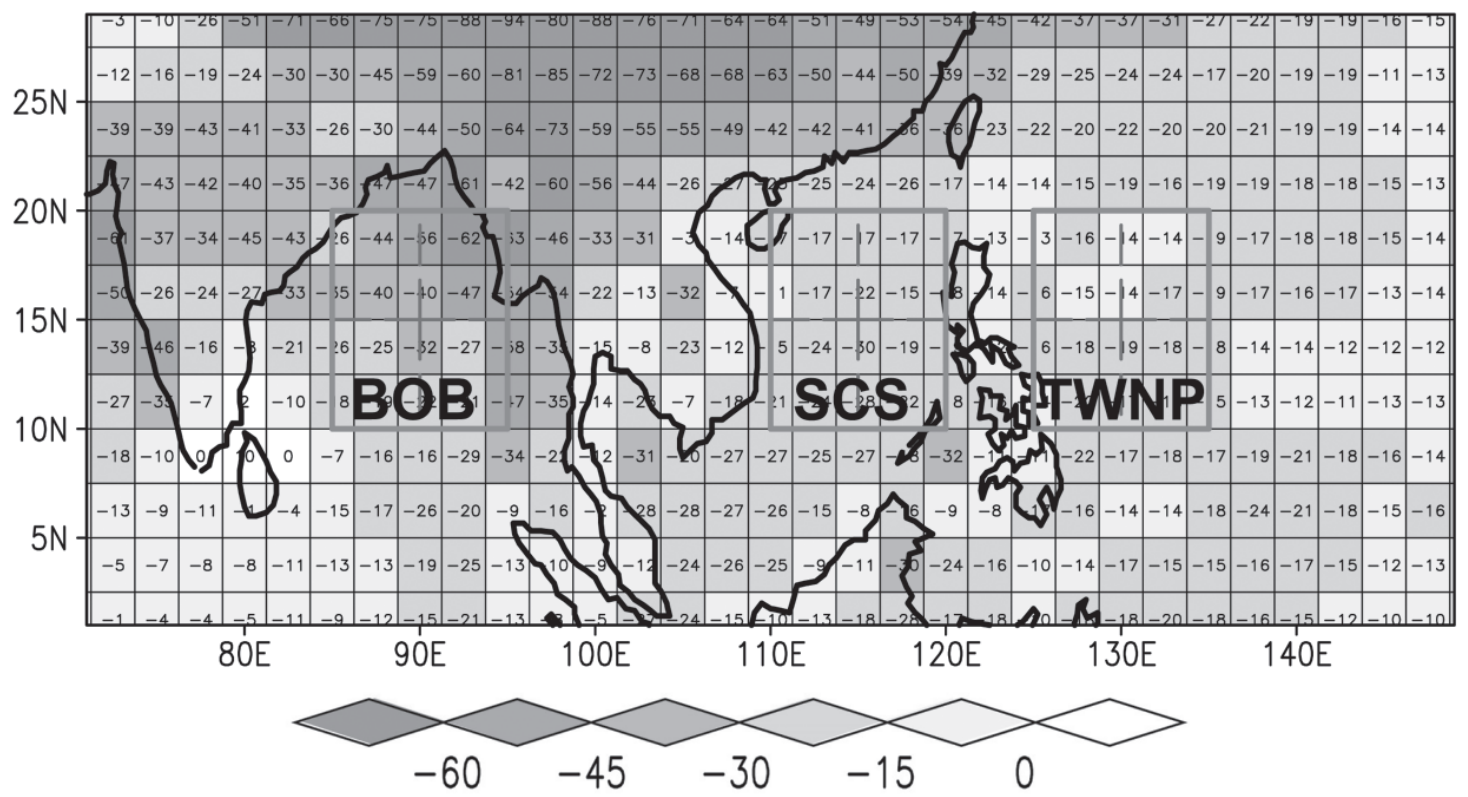

Fig. 2. The NCRF pattern (with explicit value) at the TOA during $1998 \sim 2000$ summertime (JJA). 
in mid-July in the SCS and the TWNP, which causes the magnitude of precipitation, mean cloud optical depth and CRF etc. for the entire summer to average less than that of the BOB (Kau et al. 2003). On the other hands, the mechanism generating negative NCRF in the BOB is different from that over land for China. Frequent convection in the BOB directly connects to the low-level southwesterly of the ISM system. While it is caused by deep stratus clouds, it is generated and maintained by the frictional and blocking effects of the Tibetan Plateau in China (Yu et al. 2004).

The difference between the strong NCRF in the BOB and relatively weak NCRF in the SCS and the TWNP is an important characteristic of the APSM. Figure 3 shows the relation-

(a) $\mathrm{BOB}$
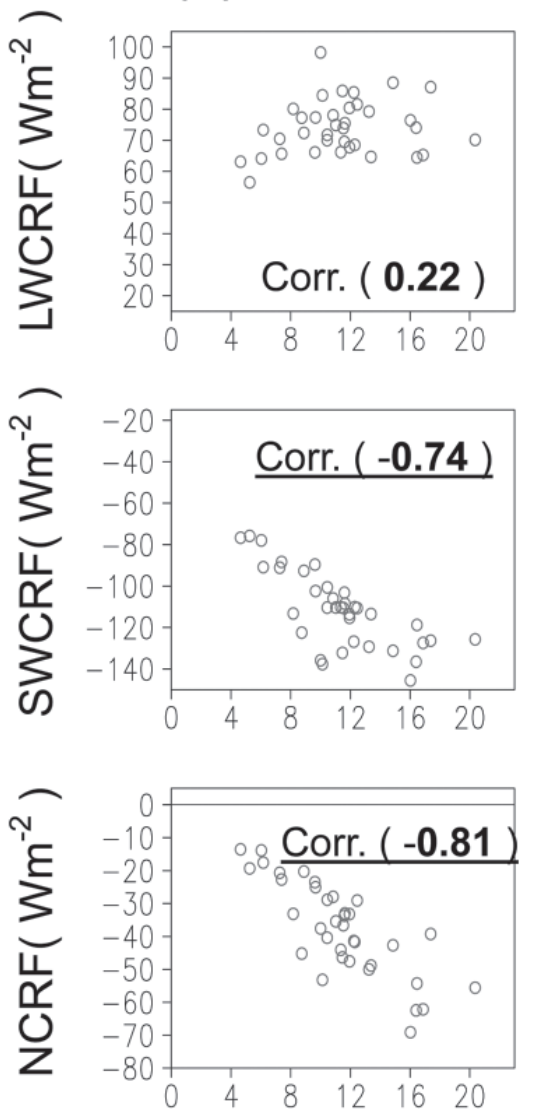

Prec. ( $\mathrm{mm} /$ day ) (b) SCS
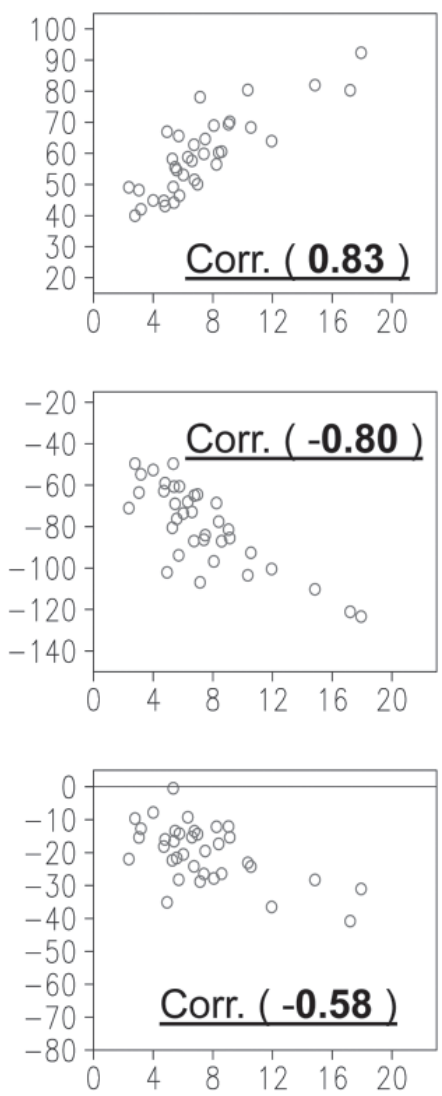

Prec. ( $\mathrm{mm} /$ day ) (c) TWNP
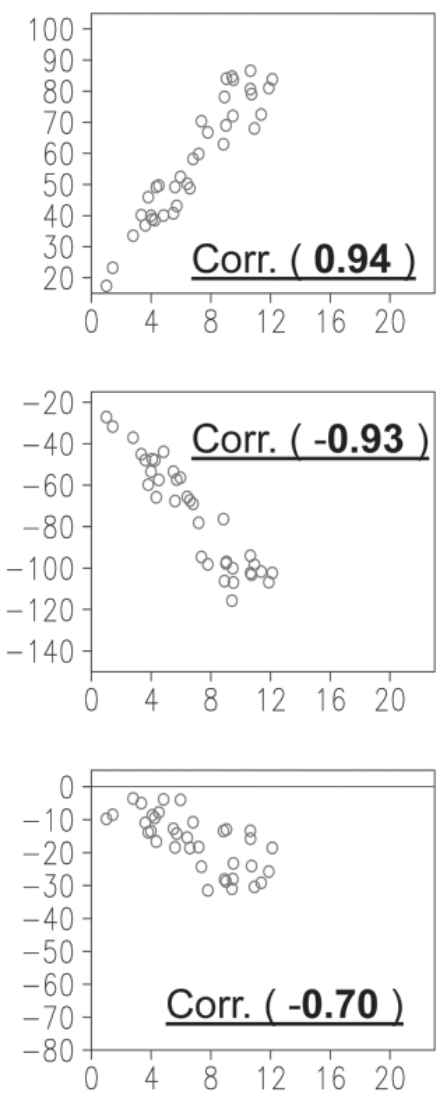

Prec. ( mm/day )

Fig. 3. Relationship between LWCRF, SWCRF, NCRF, and precipitation through monthly-mean and area-mean $\left(5^{\circ} \times 5^{\circ}\right)$ in the BOB, SCS, and TWNP (underlying correlation coefficients are significant at a 95\% level). 
ship between LWCRF, SWCRF, NCRF, and precipitation (underlying correlation coefficients are significant at a 95\% level). As shown in Fig. 3, there is a wide range of LWCRF and SWCRF in both the TWNP and SCS. This associates with better relationships between LWCRF, SWCRF, and precipitation in the SCS and TWNP than in the BOB. Through a wide range of $\mathrm{CRF}$ and a good relationship between $\mathrm{CRF}$ and precipitation, we define three categories from area-mean and daily-mean precipitation data to connect different CRF stages: heavy precipitation is defined as precipitation larger than average in summer (BOB: $11.32 \mathrm{~mm} \mathrm{day}^{-1}$; SCS: $7.36 \mathrm{~mm}_{\text {day }}{ }^{-1}$; TWNP: $6.96 \mathrm{~mm} \mathrm{day}^{-1}$ ). The percentage in time series for each region is $50 \%$ in the BOB, $38 \%$ in the SCS, and 39\% in the TWNP; medium precipitation is defined as precipitation between average and one standard deviation under the average (BOB: $11.32 \sim$ $5.11 \mathrm{~mm}$ day $^{-1}$; SCS: $7.36 \sim 0.88 \mathrm{~mm}_{\text {day }}{ }^{-1}$; TWNP: $\left.6.96 \sim 0.26 \mathrm{~mm}_{\text {day }}{ }^{-1}\right)$. The percentage of medium precipitation is 30\% in the BOB, 52\% in the SCS, and 56\% in the TWNP; and small precipitation which is defined as precipitation less than one standard deviation under the average. The percentage of small precipitation is $20 \%$ in the BOB, $10 \%$ in the SCS, and $5 \%$ in the TWNP.

Based on classification from the strength of precipitation, Fig. 4 shows an x-y scatter diagram between mean cloud optical depth and OLR (medium and small precipitation have been combined). As shown in Fig. 4, the cloud optical depth is large with heavy precipitation and is clearly defined by the classification of precipitation. The OLR is generally low because of large amount of high clouds accompanied by convection. Thus a significant SWCRF relates to large cloud optical depth, lower OLR and heavy precipitation (strong latent heating) can be defined in a general stage associated with convective clouds. On the other hand, cloud optical depth is small (except for parts of the BOB) during medium to small precipitation. However there is a wide range of OLR in each APSM region. This condition in time evolution forms another stage such that the role of CRF maybe as important as latent-heat release. The LWCRF in this stage is more important than SWCRF. The concept of two stages separating from CRF relates to high-thick and high-thin clouds, which are major cloud types over the APSM regions. The entire summer season, which is affected by different CRF, is dominated by these two stages in time evolution.

Figure 5 shows mean cloud top temperature and precipitation (minus the average in summer) in time series. We can find a good relationship between these two parameters (the correlation coefficient in three APSM regions are stronger than -0.9). The strength and tendency for heavy precipitation and low cloud top temperature are consistent. However in conditions of medium to small precipitation, cloud top temperature and precipitation are not consistent. For example (a) in the BOB, precipitation is small but cloud top temperature is near the average for August 1998 and June 1999; this maybe due to convection. Stronger convection with small coverage is comparable to the larger spatial size with relatively weaker convection typically associated with medium precipitation. These two types of cloud distribution represent different CRF and lead to a different tendency and amplitude in terms of the conditions for medium to small precipitation; and (b) in the TWNP, the precipitation decreases in mid-July 2000 but the cloud top temperature subsequently increases; this maybe due to the properties of high-thin clouds such as cirrus clouds referred by Liou (1986) and Luo and Rossow (2004). We will discuss the role of high-thin clouds in conditions of medium to small precipitation in the next section. 
In the scheme of things, the reasons for separating the stages of medium and small precipitation from the APSM time evolution, affected by convective systems, are as follows: (a) CRF in these stages maybe as important as latent-heat release for the heavy-precipitation stage; and (b) for the most part, NCRF in these stages is dominated by optically-thin high clouds. This could be a modulator for a changing climate. In the next section, we will discuss the potential role of these stages through high-cloud types with different cloud optical depth.

\section{THE ROLE OF CLOUD RADIATIVE FORCING IN THE ASIAN-PACIFIC SUM- MER MONSOON}

Based on the classification of precipitation (heavy precipitation and medium + small precipitation), Fig. 6 shows the relationship between cloud amounts of optically-thick clouds (DC + CS) and cloud amounts of optically-thin clouds (CI) (the cloud classification is defined by ISCCP

(a)

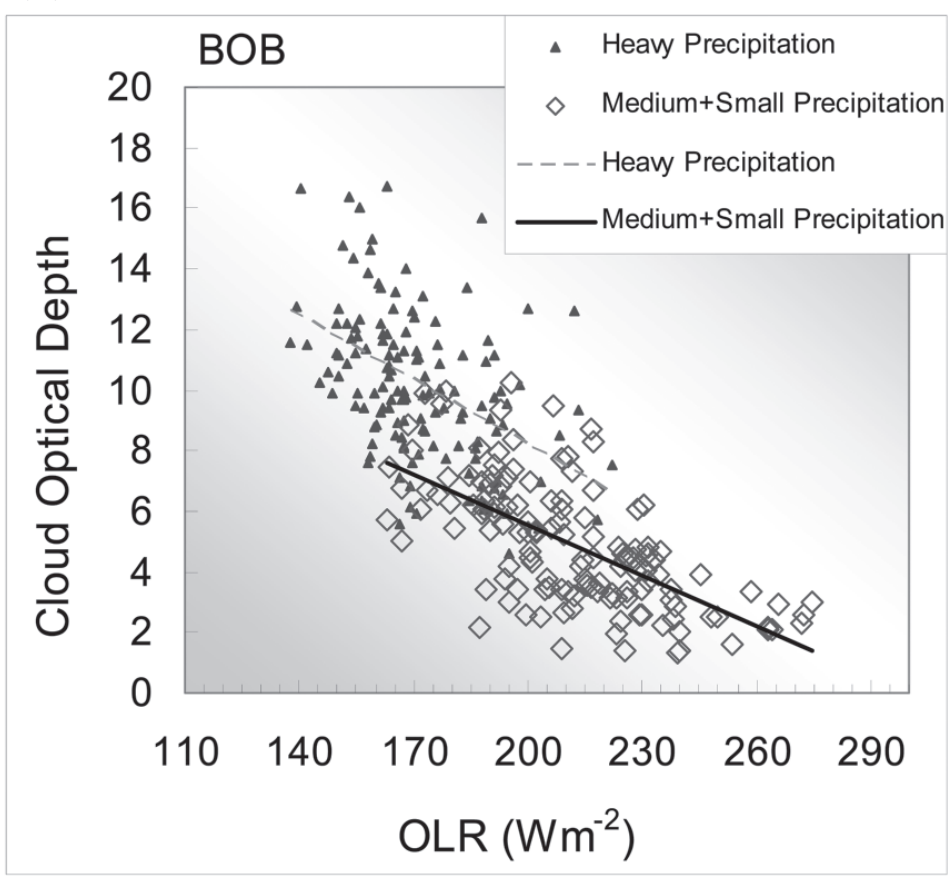

Fig. 4. Area-mean x-y scatter diagram (BOB, SCS, and TWNP) during 1998 2000 summertime between cloud optical depth and OLR. The symbol and $\diamond$ denotes heavy precipitation and medium to small precipitation respectively. 
(b)

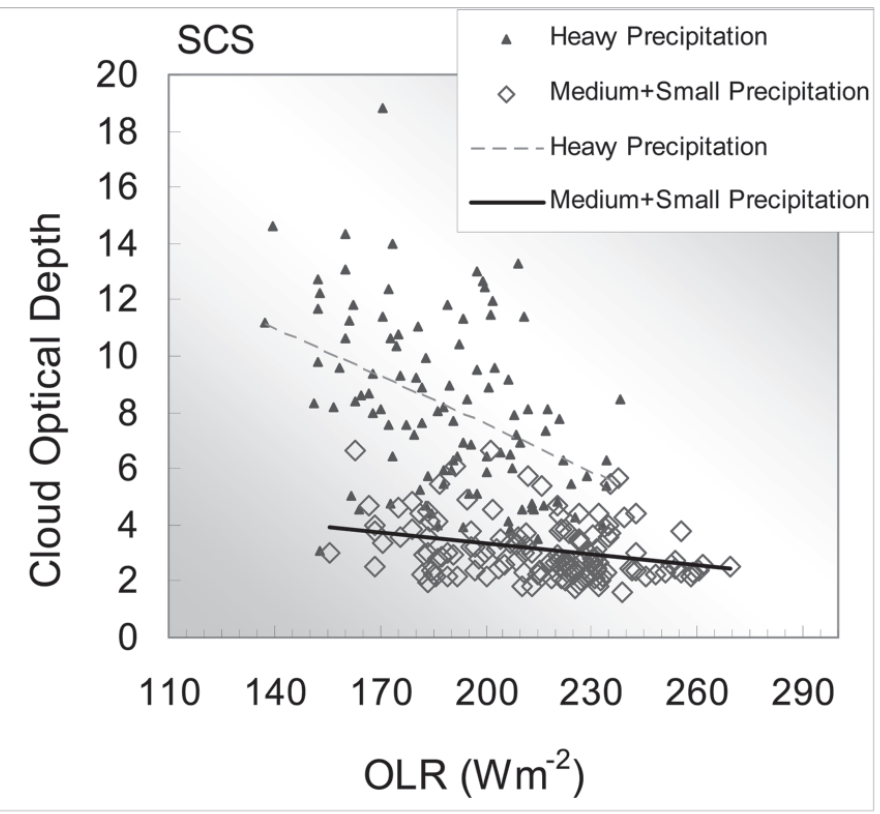

(c)

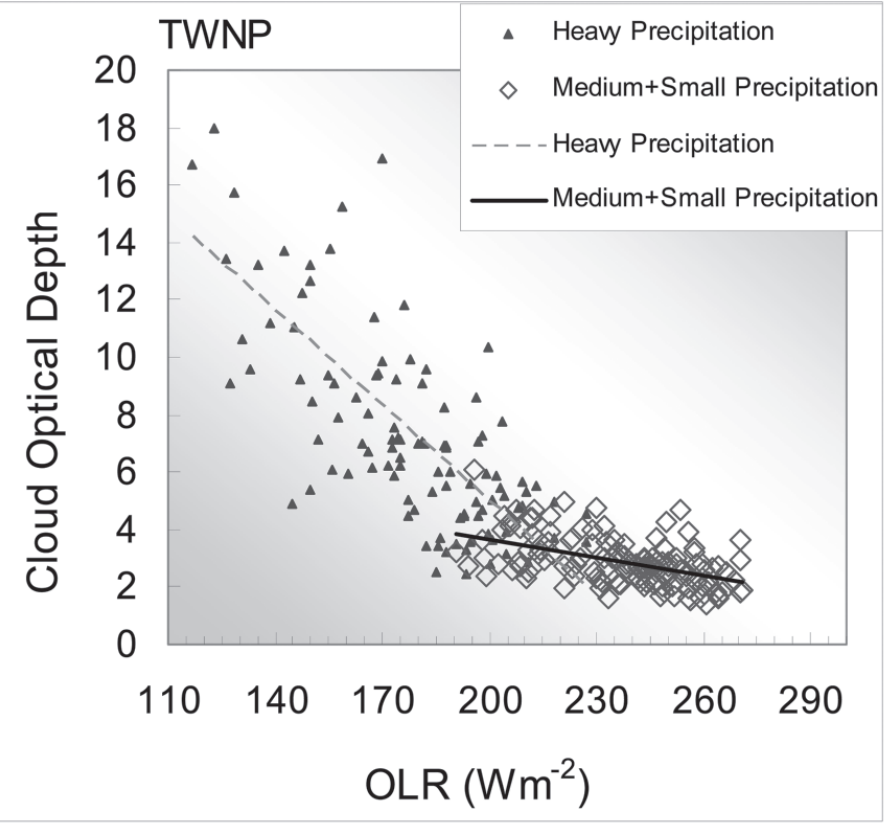

Fig. 4. (Continued) 

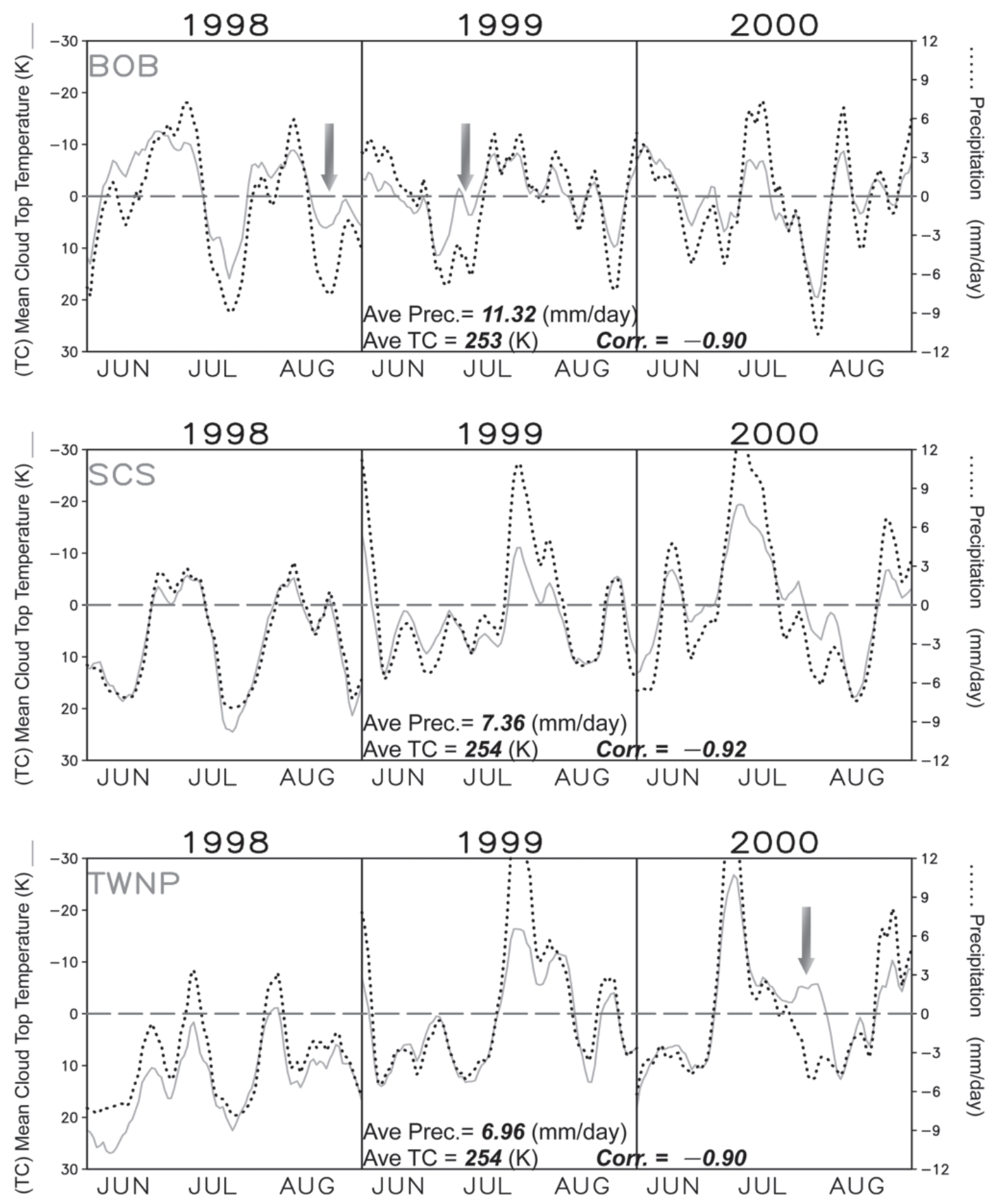

Fig. 5. Mean cloud top temperature (orange line) and precipitation (dot line) (minus summer average) of three regions (BOB, SCS, and TWNP) in time series $(1998 \sim 2000)$, see detail in the text. 
(a)

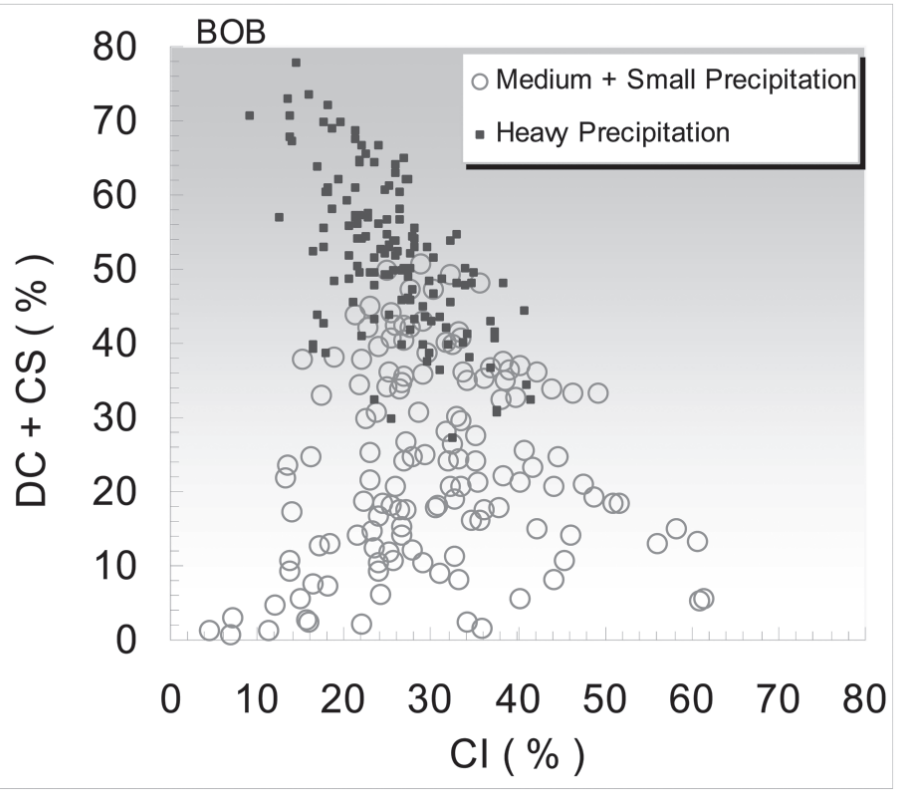

(b)

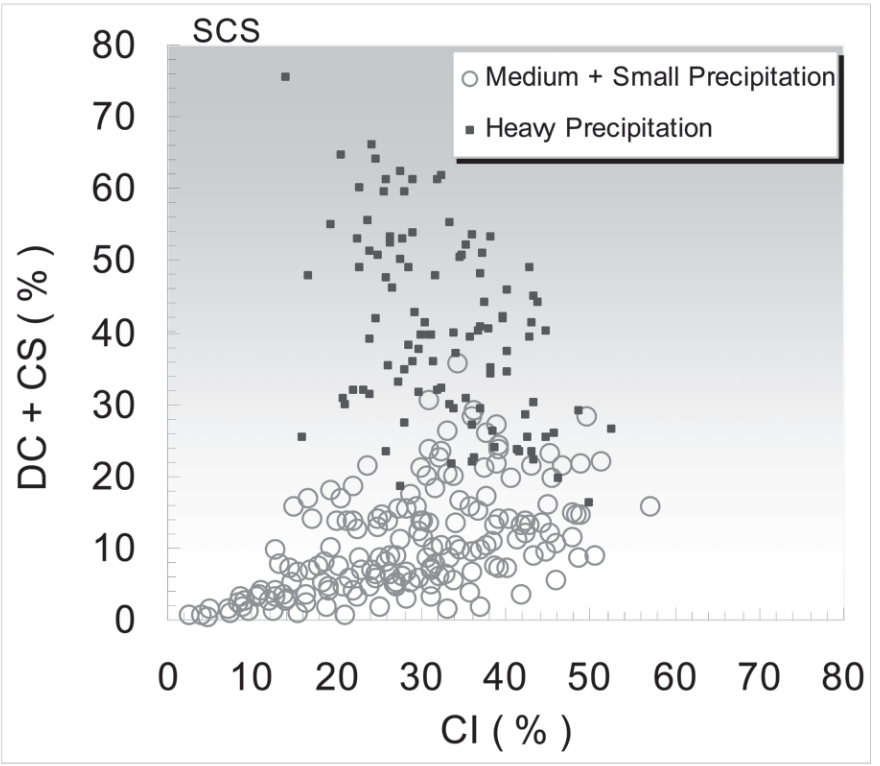

Fig. 6. Area-mean x-y scatter diagram (BOB, SCS, and TWNP) during 1998 2000 summertime between the cloud amount of DC + CS and CI. The symbol $\square$ and $\bigcirc$ denotes heavy precipitation and medium to small precipitation, respectively. 
(c)



Fig. 6. (Continued)

in Fig. 1). As shown in Fig. 6, heavy precipitation relates to cloud amounts of DC + CS. This is generally owing to the mature stage of mesoscale convection. The correlation coefficient between cloud amount of DC and CS is 0.9 in each region (not shown here). However the correlation coefficient between cloud-amount for DC + CS and CI is negative (-0.62 in BOB, -0.45 in SCS, and -0.61 in TWNP) as it is for heavy precipitation. This may result from different life cycles for DC, CS, and CI formations. On the other hand, in the stage of medium to small precipitation stages, some portion of the cloud-amount for CI may also be supported by DC and CS. While there is a wide range of cloud-amount for CI, the cloud-amount for DC + CS is obviously smaller than in heavy precipitation. The CRF during medium to small precipitation would modulate large negative NCRF during heavy-precipitation stage, and during the whole summer.

Properties such as precipitation and NCRF at the TOA may be similar during heavy precipitation (a typical convective property) in each region. The major difference between the BOB, SCS, and TWNP occurs during medium to small precipitation. Figure 7 shows cloud amounts for each cloud type based on the ISCCP cloud classification (Fig. 1) for each stage of precipitation. The percentage in the cloud-type block of DC, CS, and CI indicates mean cloud amounts of each cloud type. Through Fig. 7, we draw the following conclusions: 


\section{Cloud Amount}
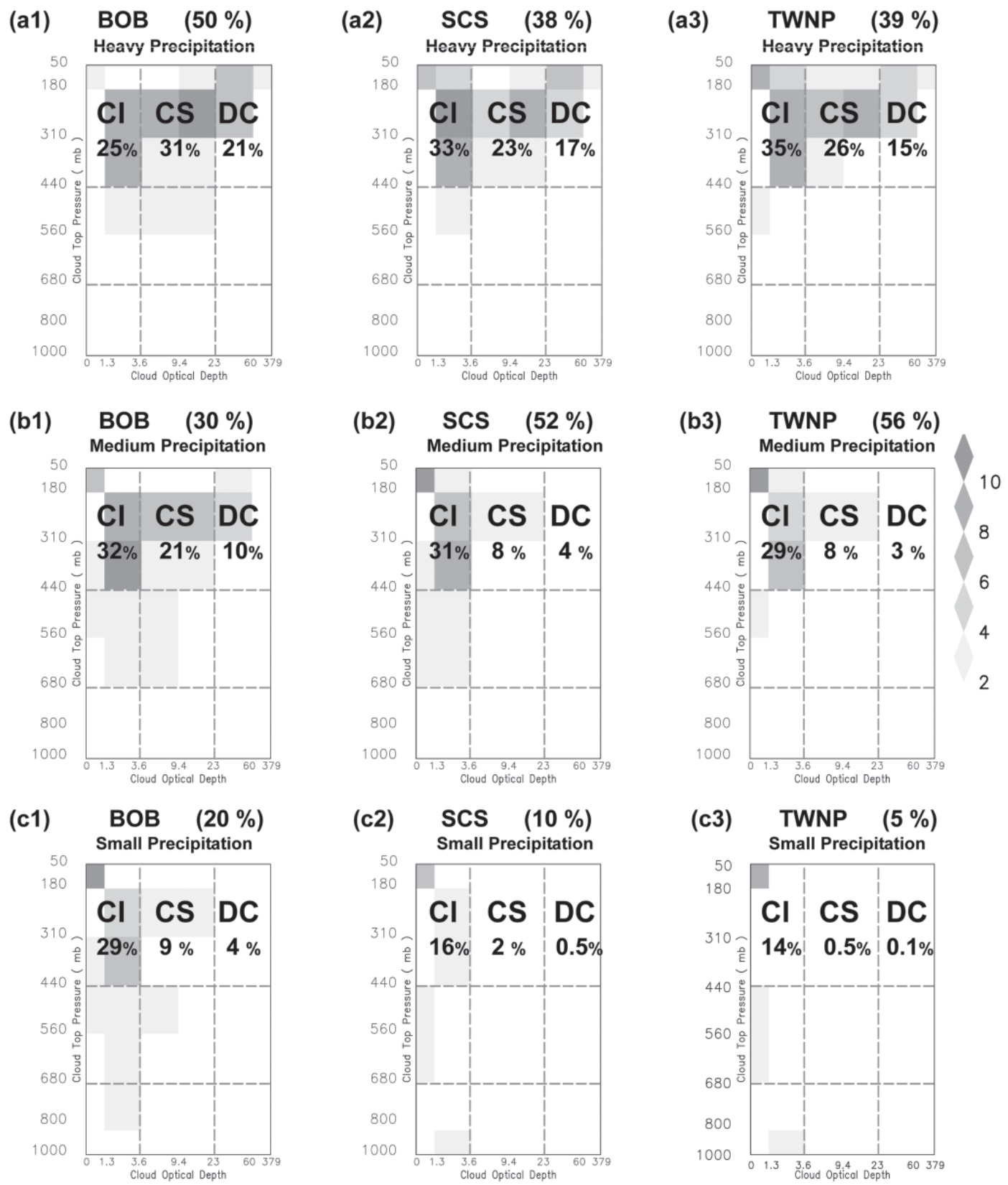

Fig. 7. Cloud amounts for each cloud type from the ISCCP cloud classification (based on Fig. 1) in each stage of precipitation. The percentage in the cloud-type block of DC, CS, and CI indicates mean cloud amounts of each cloud type, see detail in the text. 
(1) More than $80 \%$ of summertime evolutions in the BOB, SCS, and TWNP are dominated by heavy and medium precipitation (Figs. 7a, b). As shown in Fig. 7a, large cloud amounts of DC, CS, and CI cover cloudy skies by more than $75 \%(\mathrm{DC}+\mathrm{CS}+\mathrm{CI})$. This is a major convective property during the APSM period.

(2) During medium precipitation (Fig. 7b), cloud-amount for DC and CS is less than in the heavy-precipitation stage (Fig. 7a). However the cloud amount of CI is large and causes more LWCRF than SWCRF. This will modify the NCRF in this stage of medium precipitation, and be influential over the whole summer.

(3) As shown in Fig. 7c, small precipitation is caused by a small cloud-amount for DC and CS type clouds, while the cloud amount of CI is more than $10 \%$. Consequently, LWCRF should be also important during this stage.

(4) Compared with heavy precipitation, different components of high clouds are found during medium to small precipitation between three APSM regions. In the BOB, significant amounts of DC and CS clouds cause negative NCRF during medium to small precipitation. While in the SCS and TWNP, most medium to small precipitation is dominated by large amounts of CI.

Moreover, for analysis of temporal variations of cloud properties, Fig. 8 shows the spectral analysis of mean cloud optical depth from May to September. We also plot the corresponding values (thick line) at the 95 percent confidence level. As shown in Fig. 8, we can find that over the $\mathrm{BOB}$, the highest power is in the period around the intra-seasonal timescale. While over the TWNP, the leading spectral peaks are less than 10 days. The significant difference for leading spectral peaks between the BOB and TWNP could be directly related to differential properties in the medium and small precipitation stages.

\section{00 May Sep (Spectral Analysis) Cloud Optical Depth}
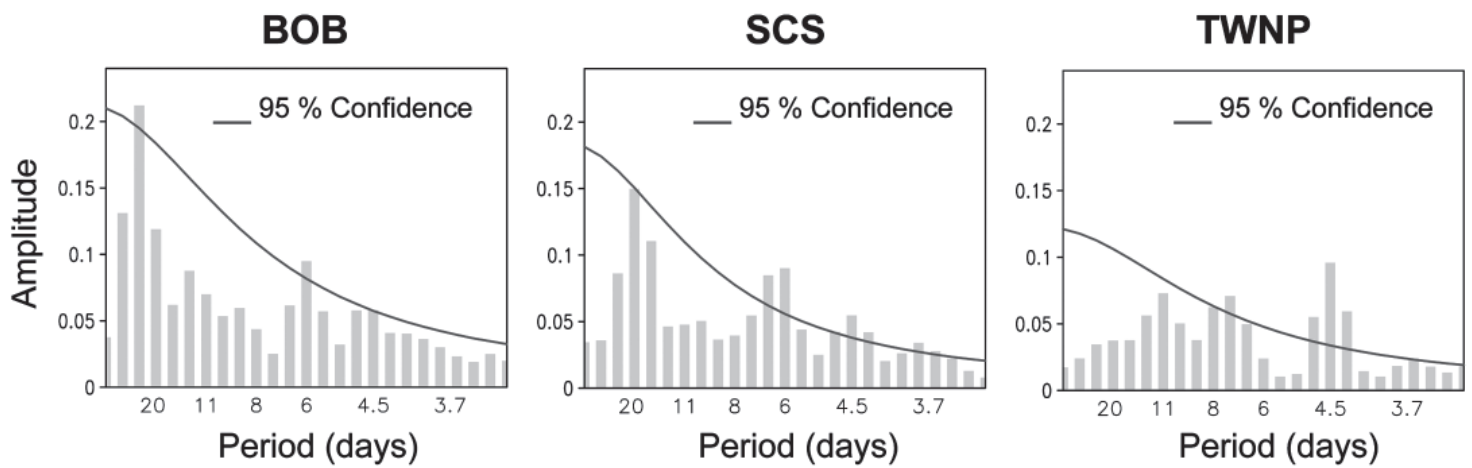

Fig. 8. Spectral analysis of mean cloud optical depth from May to September (1998 2000). The thick line denotes the corresponding values at the 95\% confidence level. 
Since the CRF of DC + CS and CI are different and are consistent in different stages of precipitation, we define a cloud-amount index from the cloud-amount of CI minus (DC + CS) to represent different CRF stages in the APSM regions. In Fig. 9, we use area-mean data to show time evolution of the cloud-amount index (shading bar) and vertical velocity (dot line indicates upward motion in -6 and $-12 \mathrm{hPa} \mathrm{s}^{-1}$ contour value). We also plot heavy precipitation and medium to small precipitation in a bar chart at the bottom of each picture. As shown in Fig. 9, a good relationship can be found between the cloud-amount index and precipitation (correlation coefficients are stronger than -0.8 in the BOB, SCS, and TWNP). Large-scale upper motion is consistent in convective properties such as during heavy precipitation and a negative cloud-amount index (dominated by DC and CS).

However, there are some inconsistencies between the cloud-amount index and precipitation stages. For example in the summer of 2000 in the TWNP, the cloud-amount for CI is larger than DC $+\mathrm{CS}$, but it is during a period of heavy precipitation and with significant upper motion at the same time. The CI cloud effect should also be important for example in this case. Although the CI cloud effect is difficult to distinguish from convective systems, it could be a modulator for a convective system and related to the difference between the BOB, SCS, and TWNP. Briefly, the CI can play a critical role in different CRF stages (based on the cloudamount index), and could play a potential role in the broad range of frequency between the APSM regions.

\section{DISCUSSION}

Information pertaining to cloud cover is the major focus of this study. Cloud-overlapping uncertainty in cloud observation is generally serious and often underestimates the effect of middle and low-level clouds. Recently, Chang and $\mathrm{Li}$ (2005) referred to a novel retrieval method that takes full advantage of satellite data from the Moderate Resolution Imaging Spectroradiometer (MODIS). They can identify overlapping high cirrus and low water clouds more accurately. This is helpful in discussing middle and low-level clouds, especially in terms of shallow convection in the APSM regions.

Cloud feedback into the climate does not follow a single pathway [see Stephens (2005) for a critical review of cloud-climate feedbacks mechanisms]. There needs to be more detailed analysis of cloud feedback, especially during stages affected by CI clouds. Compared to the effects of DC clouds, most sea surface temperatures (SSTs) increase when the CI clouds dominate (not shown). However, CI may also have a kind of "thermostat" mechanism whereby there is an increase in thick CI in response to an increase in SST. This will reduce solar heating and limit SST to observed high values (Ramanathan and Collins 1991). Whether CI can cause limited SSTs or not, this portion of CI clouds indicates highly reflective CI detrained from convection. We have explored the relationship between different CI types in the ISCCP cloud classification (two sub-CI types separated from cloud optical depth are shown in Fig. 1). The result shows that not all CI clouds have a good relationship with convection in cloudy regions. We shall perform further analyses of CI cloud effects especially in terms of extensive cloud amounts separating from the DC clouds in the future. 

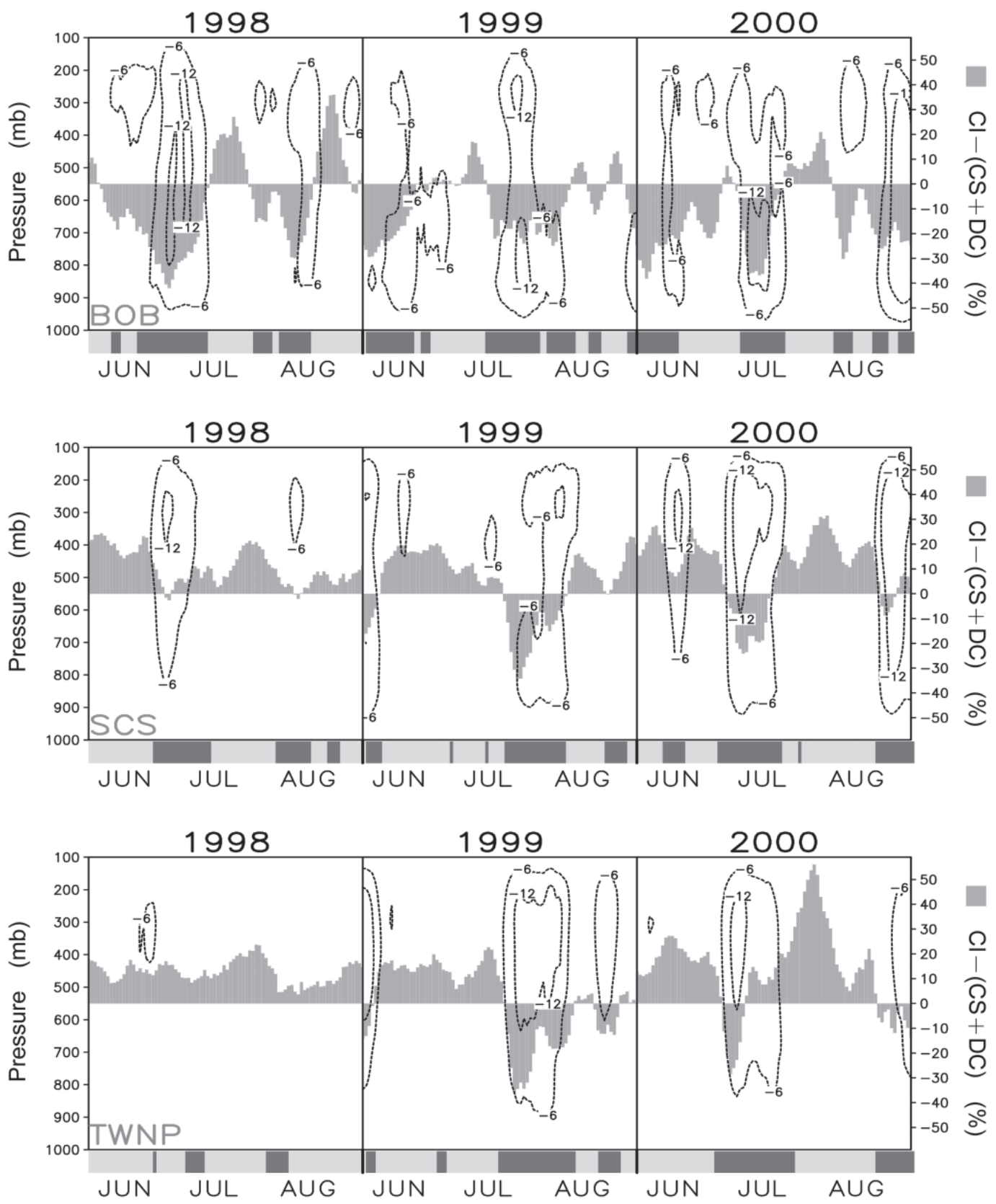

Heavy Precipitation , Medium + Small Precipitation

Fig. 9. The cloud-amount index (cloud amount of DC + CS minus CI, orange shading) and vertical velocity in each level (thick contour). The bar chart at the bottom of each picture denotes heavy precipitation and medium to small precipitation respectively. 
Another critical issue requiring discussion is that of GCMs being in general forced by observed SSTs, and changes in convective systems being intrinsically linked to SST distribution. However, observed cloud forcing and its variability is the result of interaction between the atmosphere and the ocean, so its variability provides an important means of improving climate models, especially for the couple model. Allan et al. (2002) also asked this question. They noted that without accurate information for convective systems or their effects, accurate simulation of cloud feedback mechanisms remains in question. Our concern in this study is to have correct and meaningful observed SST (to have correct cloud information) to force GCM. The different stages for example for separating CRF by using a cloud-amount index ought be more helpful in present GCMs for dealing with the effects from more than two cloud types associated with convective systems. Moreover, the nocturnal rainfall which derived from deeper convective cells (Sui et al. 1997) should be important resulting in different stages of CRF, and potentially in modulating atmospheric low-frequency variability (Lau and Sui 1997).

\section{SUMMARY}

In this study, we analyze the role of CRF in the APSM. We define two stages based on strength of area-mean precipitation to connect different clouds and their CRF in time evolution. Comparing stages with their respective significant properties in convectively active regimes, $\mathrm{CRF}$ appears to be as important as latent-heat release during medium to small precipitation. Large amount of CI clouds and their CRF play a critical role in the stage of medium to small precipitation. $\mathrm{CI}$ in this stage could be a modulator for a changing climate. The major difference between the APSM regions (the BOB, SCS, and TWNP) occurs in this stage.

Convective-cloud clusters with strong precipitation occur most frequently in the BOB, SCS, and TWNP during the APSM period. Heavy precipitation in general accompanies significant convective properties an observation that is similar amongst all APSM regions. However, expansive convective-cloud clusters and differential frequency in convective systems cause difference between the APSM regions. In the BOB, SCS, and TWNP, greater than half of the time evolution belongs to medium to small precipitation. There is more than one possibility for the formation of this complex stage; such as strong convection with smaller spatial size or weak convection with larger spatial size. Both possibilities cause different CRF through their large-scale cloud distributions.

In the BOB, significant amount of optically-thick clouds (DC and CS) cause negative NCRF during medium to small precipitation, while the SCS and TWNP is dominated by large amounts of CI clouds in this stage. This could be the cause of such a broad range of frequency between the APSM regions. For example the highest power of cloud optical depth in the BOB is in the period around the intra-seasonal timescale, while over the TWNP the leading spectral peaks are less than 10 days. The result may reflect the work of Metha and Smith (1997), who used a one-dimensional radiative-convective equilibrium model to estimate the influence of the radiative damping timescale. They hypothesized that variable cloud-radiative cooling rates can alter phase speeds of Kelvin waves. In the BOB, large negative NCRF may be associated with variability in low-frequency oscillation. The positive NCRF caused by CI during medium 
to small precipitation suppresses the NCRF for the whole of summer and cause different frequency in the TWNP.

Based on three types of high clouds, we find a useful cloud-amount index [cloud amount of CI minus (DC $+\mathrm{CS})$ ] to effectively represent two CRF stages in time series for the APSM. The correlation coefficients between the cloud-amount index and precipitation are stronger than -0.8 in each APSM region. For GCMs, the poor simulation of intraseasonal variability may be associated with the inability to simulate the role of CI clouds accurately. It is important to have correct cloud information for better cloud-SST variability (for the process of cloud forcing to have more meaningful fixed-observed SST to force the GCMs). Therefore, the concept of separating meaningful CRF stages from the APSM time evolution ought to be more appropriate for APSM studies and simulation than having just one cloud effect in GCMs.

Acknowledgements The authors would like to thank anonymous reviewers for their sugguestions and valuable comments greatly improved the manuscript. This research was supported by the National Science Council of Taiwan under grant NSC 94-2111-M-002-011-AP4 and NSC 95-2111-M-002-020-AP4.

\section{REFERENCES}

Allan, R. P., A. Slingo, and M. A. Ringer, 2002: Influence of dynamics on the changes in tropical cloud radiative forcing during the 1998 El Niño. J. Climate, 15, 1979-1986.

Briegel, L. M., and W. M. Frank, 1997: Large-scale influences on tropical cyclogenesis in the western North Pacific. Mon. Weather Rev., 125, 1397-1413.

Chang, F. L., and Z. Li, 2005: A new method for detection of cirrus overlapping water clouds and determination of their optical properties. J. Atmos. Sci., 62, 3993-4009.

Cox, S. K., 1971: Cirrus clouds and the climate. J. Climate, 28, 1513-1515.

Frank, W. M., 1982: Large-scale characteristics of tropical cyclones. Mon. Weather Rev., 110, $572-586$.

Fu, R., A. Del Genio, and W. B. Rossow, 1990: Behavior of the convective clouds in the tropical Pacific deduced from ISCCP radiances. J. Climate, 3, 1129-1152.

Hartmann, D. L., and D. Doelling, 1991: On the net radiative effectiveness of clouds. $J$. Geophys. Res., 96, 869-891.

Houze, R. A., Jr., 1993: Cloud Dynamics. Academic Press, 576 pp.

Houze, R. A., Jr., 2004: Mesoscale convective systems. Rev. Geophys., 42, 1-43.

Jakob, C., 2003: An improved strategy for the evaluation of cloud parameterizations in GCMs. Bull. Amer. Meteorol. Soc., 84, 1387-1401.

Kau, W. S., C. H. Wu, and C. H. Tsou, 2003: The cloud radiative forcing over Asian-Pacific summer monsoon region. Terr. Atmos. Ocean. Sci., 14, 445-467.

Kummerow, C., W. Barnes, T. Kozu, J. Shiue, and J. Simpson, 1998: The Tropical Rainfall Measuring Mission (TRMM) sensor package. J. Atmos. Ocean. Technol., 15, 809-817. 
Lander, M. A., 1994: Description of a monsoon gyre and its effects on the tropical cyclones in the western North Pacific during August 1991. Weather Forcast., 9, 640-654.

Lau, K. M., and C. H. Sui, 1997: Mechanisms of short-term sea surface temperature regulation: Observations during TOGA COARE. J. Climate, 10, 465-472.

Lin, H., and B. Wang, 2002: The time-space structure of Asian summer monsoon - a fast annual cycle view. J. Climate, 15, 2001-2019.

Liou, K. N., 1986: Influence of cirrus clouds on weather and climate processes: A global perspective. Mon. Weather Rev., 114, 1167-1199.

Luo, Z., and W. B. Rossow, 2004: Characterizing tropical cirrus life cycle, evolution, and interaction with upper-tropospheric water vapor using Lagrangian trajectory analysis of satellite observations. J. Climate, 17, 4541-4563.

Ma, C. C., C. R. Mechoso, A. W. Robertson, and A. Arakawa, 1996: Peruvian stratus clouds and the tropical Pacific circulation: A coupled ocean-atmosphere GCM study. J. Climate, 9, 1635-1645.

Mapes, B. E., and J. R. A. Houze, 1993: Cloud clusters and superclusters over the oceanic warm pool. Mon. Weather Rev., 121, 1398-2037.

Metha, A., and E. A. Smith, 1997: Variability of radiative cooling during the Asian summer monsoon and its influence on Intraseasonal waves. J. Atmos. Sci., 54, 941-966.

Rajeevan, M., and J. Srinivasan, 2000: Net cloud radiative forcing at the top of atmosphere in the Asian monsoon region. J. Climate, 13, 650-657.

Ramanathan, V., E. J. Pitcher, R. C. Malone, and M. L. Blackmon, 1983: The response of a spectral general circulation model to refinements in radiative processes. J. Atmos. Sci., 40, 605-630.

Ramanathan, V., and W. Collins, 1991: Thermodynamic regulation of ocean warming by cirrus clouds deduced from observations of the 1987 El Niño. Nature, 351, 27-32.

Randall, D. A., Harshvardham, D. A. Dazlich, and T. G. Corsetti, 1989: Interactions among radiation, convection, and large-scale dynamics in a general circulation model. J. Atmos. Sci., 46, 1943-1970.

Ritchie, E. A., and G. J. Holland, 1999: Large-scale patterns associated with tropical cyclogenesis in the western Pacific. Mon. Weather Rev., 127, 2027-2034.

Rossow, W. B., and L. C. Garder, 1993: Cloud detection using satellite measurements of infrared and visible radiances for ISCCP. J. Climate, 5, 2341-2369.

Rossow, W. B., and R. A. Schiffer, 1999: Advances in understanding clouds from ISCCP. Bull. Amer. Meteorol. Soc., 80, 2261-2288.

Rossow, W. B., and E. N. Dueñas, 2004: The international satellite cloud climatology project (ISCCP) web site: An online resource for research. Bull. Amer. Meteorol. Soc., 85, 167-172.

Slingo, A., and J. M. Slingo, 1988: The response of a general circulation model to cloud longwave radiative forcing. I: Introduction and initial experiments. Q.J.R. Meteorol. Soc., 114, 1027-1062.

Slingo, A., and J. M. Slingo, 1991: Response of the national center for atmospheric research community climate model to improvements in the representation of clouds. J. Geophys. Res., 96, 15341-15357. 
Stephens, G. L., 2005: Review article: Cloud feedbacks in the climate system: A critical review. J. Climate, 18, 237-273.

Sui, C. H., K. M. Lau, Y. N. Takayabu, and D. A. Short, 1997: Diurnal variations in tropical oceanic cumulus convection during TOGA COARE. J. Atmos. Sci., 54, 639-655.

Wang, B., and H. Lin, 2002: Rainy season of the Asian-Pacific summer monsoon. J. Climate, 15, 386-396.

Yu, R., B. Wang, and T. Zhou, 2004: Climate effects of the deep continental stratus clouds generated by the Tibetan Plateau. J. Climate, 17, 2702-2713.

Wu, C. H., and W. S. Kau, 2007: The role of cloud radiative forcing in the Asian-Pacific summer monsoon. Terr. Atmos. Ocean. Sci., 18, 623-642, doi: 10.3319/TAO.2007.18. 3.623(A). 